\title{
HIPERTENSÃO ARTERIAL NA DOENÇA RENAL CRÔNICA EM PEQUENOS ANIMAIS - REVISÃO DA LITERATURA
}

\author{
GALVÃO, André Luiz Baptista ${ }^{1}$ \\ BORGES, Juliana Corrêa ${ }^{2}$ \\ VIEIRA, Manuela Cristina ${ }^{1}$ \\ FERREIRA, Guadalupe Sampaio ${ }^{3}$ \\ LÉGA, Elzylene ${ }^{4}$ \\ PINTO, Mildre Loraine ${ }^{5}$
}

\begin{abstract}
RESUMO: A doença renal crônica (DRC) é caracterizada por lesões estruturais irreversíveis, que podem evoluir progressivamente para uremia e falência renal. Após a instalação inicial da lesão renal, ocorrem mudanças estruturais e funcionais adaptativas dos néfrons remanescentes, na tentativa de manter a homeostase, principalmente quanto à regulação do volume e da composição do fluido corporal extracelular. A hipertensão sistêmica é uma das complicações cardiovasculares mais frequentemente reconhecidas da DRC em cães e gatos. Os órgãos mais afetados pela hipertensão são os que estão envolvidos na regulação do fluxo sanguíneo (coração) e os que contêm extensas redes arteriolares ou capilares (olhos, cérebro, rins). Nos rins a hipertensão esta associada a presença de proteinúria e progressão de dano glomerular. Desse modo, apresentamos esta revisão de literatura com objetivo de descrever as alterações sistêmicas relacionadas com o envolvimento da doença renal crônica com a hipertensão arterial, como também sua patofisiologia, recursos em diagnóstico, condutas e opções terapêuticas a serem adotadas com o paciente.
\end{abstract}

Palavra-chaves: Cão. Gato. Doença Renal Crônica. Hipertensão.

\section{ARTERIAL HYPERTENSION IN THE CHORNIC KIDNEY DISEASE IN SMALL ANIMALS - REVIEW}

SUMMARY: Chronic kidney disease (CKD) is characterized by irreversible structural lesions can develop progressively for uremia and chronic renal failure (CRF). After first renal lesion, occur structural and functional alterations in remaining nephrons, with objective to maintain homeostasis, mainly for the volume and the composition fluid extracell. Systemic hypertension is the most important cardiovascular complications in CKD in the dogs and cats. Organs more affected by hypertension are these involved in the regulation sanguine flow (heart) and others that contain extensive arteriolares or capillary nets (eyes, brain, kidneys). In the kidneys hypertension is associated with proteinuria presence and progression glomerular damage. This way, the objective this literary revision was to describe systemic alterations related with the involvement chronic renal disease with arterial hypertension, as well as your pathophysiology, resources in diagnosis, conducts and therapeutic options for be use in the patient.

Keywords: Cat. Dog. Chronic kidney disease. Hypertension.

\footnotetext{
${ }^{1}$ Pós-graduando em Medicina Veterinária da Faculdade de Ciências Agrárias e Veterinárias, - Universidade Estadual Paulista (FCAV/UNESP) - Jaboticabal. Via de Aceso "Prof Dr. Paulo Donato Castellane" Km 05, 14.884-900, Jaboticabal -SP. Brasil.

2 Docente do Departamento de Morfologia e Fisiologia Animal da Faculdade de Ciências Agrárias e Veterinárias, - Universidade Estadual Paulista (FCAV/UNESP) - Jaboticabal-SP

${ }^{3}$ Médica Veterinária Autônoma

${ }^{4}$ Docente e Coordenadora do Curso de Graduação em Medicina Veterinária da Faculdade Dr. Francisco Maeda FAFRAM - Fundação Educacional de Ituverava-SP.

${ }^{5}$ Docente do Setor de Diagnóstico por Imagem do Centro Universitário Barão de Mauá - Ribeirão Preto-SP.
} 


\section{INTRODUÇÃO}

O sistema urinário é de grande importância para diferentes funções orgânicas, o rim mantém ou controla os volumes do líquido extracelular e do sangue, a pressão arterial sistêmica, a produção de eritrócitos, a excreção de catabólitos nitrogenados e o equilíbrio de uma série de eletrólitos mantendo a homeostase (POLZIN et al., 2000).

A doença renal crônica (DRC) é caracterizada por lesões estruturais irreversíveis, que podem evoluir progressivamente para uremia, insuficiência renal crônica (IRC) e falência renal (POLZIN et al., 2000). Após a instalação inicial da lesão renal, ocorrem mudanças estruturais e funcionais adaptativas dos néfrons remanescentes, na tentativa de manter a homeostase, principalmente quanto à regulação do volume e da composição do fluido corporal extracelular. Eventualmente, essas mudanças adaptativas tornam-se excessivas ou ineficientes, favorecendo ainda mais, o desenvolvimento de injúria dos néfrons (POLZIN et al., 2005; SHIMIZU, 2005).

A mudança adaptativa consiste no aumento do volume de filtração glomerular e do aporte sanguíneo dos néfrons remanescentes, com consequente hipertrofia e hipertensão glomerular, na tentativa de manter a taxa de filtração glomerular (TFG), e atenuar a diminuição do clearance de creatinina, deste modo exacerba-se a agressão hemodinâmica ao glomérulo, como consequência, estabelece-se um ciclo vicioso que leva, ao final do processo, à completa destruição do parênquima renal (SHIMIZU, 2005).

Depois de estabelecida a DRC, a magnitude da disfunção renal geralmente permanece estável por meses ou declina vagarosamente no decorrer de meses a anos. Não é necessário que o processo responsável pela lesão inicial, esteja presente para que ocorra uma disfunção progressiva (JACOB, 2002).

$\mathrm{Na}$ tentativa de facilitar a instituição de prognóstico e tratamentos adequados ao grau de severidade da DRC, a International Renal Interest Society (IRIS, 2006) dividiu a mesma em estágios:

- Estágio I (não azotêmico): ausência de sintomas evidentes de uremia, exceto poliúria e polidipsia e creatinina sérica menor que $1,4 \mathrm{mg} / \mathrm{dL}$ para cães e inferior $1,6 \mathrm{mg} / \mathrm{dL}$ para gatos.

- Estágio II (azotemia renal discreta): ausência de sintomas evidentes de uremia, exceto poliúria e polidipsia e creatinina sérica entre 1,4 e 2,0mg/dL para cães e entre 1,6 e $2,8 \mathrm{mg} / \mathrm{dL}$ para gatos. 
- Estágio III (azotemia renal moderada): sintomas moderados de uremia e creatinina sérica entre 2,1 e 5,0mg/dL para cães e entre 2,9 e 5,0mg/dL para gatos.

- Estágio IV (azotemia renal severa): sintomas graves de uremia e creatinina sérica maior que $5,0 \mathrm{mg} / \mathrm{dL}$ para cães e gatos.

Dentro de cada estágio, o paciente é subcategorizado de acordo com o grau de proteinúria e pressão sanguínea arterial. Os estágios II e III incluem pacientes com IRC, onde a presença de azotemia reflete a perda de mais de dois terços de néfrons funcionais. $\mathrm{O}$ termo falência renal é aplicado aos pacientes categorizados no estágio IV (IRIS, 2006).

A hipertensão sistêmica é uma das complicações cardiovasculares mais frequentemente reconhecidas da DRC em cães e gatos, considerando-se que ocorrem em 50 a 93\% dos cães acometidos e em 65\% dos gatos (COWGILL; KALLET apud KIRK, 1986; TILLEY, 2008), sendo principalmente de origem secundária (ACIERNO e LABATO, 2005; GREGORY, 2005), ainda, a hipertensão arterial pode ser detectada em $85 \%$ dos cães com doença glomerular (BARTGES, 1996). A hipertensão arterial é definida como o aumento persistente da pressão arterial sistêmica (MORAIS; PEREIRA, 2002). A presente revisão de literatura teve como objetivo descrever as alterações sistêmicas relacionadas com o envolvimento da doença renal crônica com a hipertensão arterial, como também a sua patofisiologia, recursos em diagnóstico, condutas e opções terapêuticas a serem adotadas com o paciente doente renal crônico hipertenso.

\section{PATOFISIOLOGIA NA DOENÇA RENAL CRÔNICA}

Não se conhece o mecanismo exato etiológico da hipertensão associada a DRC, não tendo sido demonstrado nenhum fator isolado responsável pelo seu estabelecimento (DARKE et al., 2000). Provavelmente, vários fatores em combinação levam ao desenvolvimento de uma hipertensão sistêmica secundária, a saber: (1) ativação do sistema renina-angiotensinaaldosterona, assim expansão do volume do líquido extracelular; (2) aumento dos níveis séricos de epinefrina e norepinefrina; (3) aumento da resposta vascular a norepinefrina; (4) diminuição da atividade do sistema cinina-calicreína; (5) aumento do débito cardíaco; (6) vasopressina, cuja elevação ajuda a promover vasoconstrição, além de exercer efeito prolongado de reduzir a excreção hídrica pelos rins e (7) aumento da resistência periférica (ROSS, 1992; SCHIFFRIN et al., 2007).

Recentemente vários estudos de Xu et al. (2005), Schiffrin et al. (2007), Desir (2008), Schlaich et al. (2009) e Desir (2009) demonstraram um novo fator relacionado à hipertensão 
arterial presente nos pacientes renais crônicos humanos, devido a deficiência de uma amina oxidase, denominada renalase oxidase. Acredita-se, que esta enzima, seja produzida nos rins e que tenha uma participação na regulação da função cardíaca, e da pressão arterial, por atuar na degradação das catecolaminas séricas, promovendo uma diminuição da pressão arterial. Xu et al. (2005) descreveram o efeito da renalase oxidase em ratos como um agente inotrópico negativo no coração e como hipotensor. Ainda, Desir (2009) descreveu a diminuição sérica da renalase oxidase, correlacionada com uma diminuição na TFG em pacientes renais crônicos com hipertensão.

A elevação da pressão sanguínea sistêmica pode apressar a progressão da DRC (TOTO et al., 1995). A hipertensão sistêmica é identificada como um fator de risco expressivo para o desenvolvimento de insuficiência renal terminal (KLAG et al., 1996; SCHIFFRIN et al., 2007). Recentemente Jacob et al. (2003) descobriram que uma alta pressão sanguínea sistólica no momento do diagnóstico inicial da DRC em cães, é associada com um risco aumentado de ocorrência de crise urêmica, risco de morte e taxa de declínio da função renal, no seu estudo $31 \%$ dos cães apresentaram hipertensão secundária a DRC, e apresentaram mau prognóstico. A hipertensão sistêmica perpetua o dano vascular e aumenta a resistência vascular periférica e renal, a qual induz a progressão da DRC, e acentua a hipertensão arterial (STILES et al., 1994). Desta maneira, os órgãos mais afetados são os que estão envolvidos na regulação do fluxo sanguíneo (coração) e os que contêm extensas redes arteriolares ou capilares (olhos, cérebro, rins) (ACIERNO; LABATO, 2005). A hipertrofia e a fibrose do ventrículo esquerdo do coração é consequência comum da hipertensão arterial, resulta em queda da tensão da parede do miocárdio (SCHIFFRIN et al., 2007; GRAUER, 2009). Isto capacita o coração suplantar a pós-carga aumentada resultante da maior resistência periférica. Não está esclarecido o mecanismo pelo qual o estímulo hemodinâmico da sobrecarga de pressão resulta em hipertrofia ventricular (MUNAGALA et al., 2005). Outros fatores, podem também influenciar a resposta cardíaca nos casos de hipertensão arterial, como o aumento da pré-carga (sobrecarga de volume), devido à expansão do volume vascular e ao aumento da contratilidade, em decorrência da maior estimulação adrenérgica (FROHLICH, 1993). O ventrículo hipertrofiado pode estar sob maior risco de isquemia, devido às quedas relativas no fluxo sanguíneo das artérias coronárias (LONDON et al., 1996). A hipertrofia ventricular pode ser tão grave a ponto de causar disfunção cardíaca congestiva, mas se a pressão sanguínea for controlada a hipertrofia pode regredir (ROSS, 1992; MUNAGALA et al., 2005).

Estudos realizados em pacientes humanos demonstraram a existência de uma relação 
entre proteinúria e hipertensão, visto que o controle terapêutico da última está implicado em uma diminuição da magnitude da primeira (GRAUER et al., 2000; GRAUER, 2009). A correlação de hipertensão e proteinúria têm sido descrita em cães com diabetes mellitus (STRUBLE et al.,1998), em cães com hiperadrenocorticismo (ORTEGA et al., 1996), bem como em cães com DRC (REGO, 2006; WEHNER et al., 2008).

A ativação do sistema renina-angiotensina-aldosterona está diretamente relacionada com a hipertensão arterial e também provoca a proteinúria. A hipertensão capilar glomerular leva a um aumento da permeabilidade glomerular e excessiva filtração de proteínas (GRAUER, 2009). Este sistema também pode causar proteinúria através da expressão de nefrina. Esta é uma proteína transmembrana localizada nos "slits" diafragmáticos dos podócitos glomerulares. Pela manutenção da integridade destes "slits", a nefrina limita a perda de proteínas pelo glomérulo. Quando sua expressão é alterada ocorre a proteinúria. Proteínas presentes na urina são tóxicas para os túbulos e podem provocar inflamação tubulointersticial, fibrose e morte celular (GREGORY, 2005; SHIMIZU, 2005; Scott, 2008). A proteinúria pode estar presente como consequência de lesões glomerulares e/ou tubulares (GREGORY, 2005; GRAUER, 2009). Usualmente, proteinúria de maior intensidade é observada nas doenças glomerulares. As doenças túbulos-intersticiais que evoluem para DRC apresentam proteinúria, de intensidade discreta ou até mesmo ausente (FINCO, 1995; GRAUER, 2009).

A proteinúria de origem renal é decorrente de alterações estruturais na barreira glomerular, que causam a passagem de uma quantidade maior de proteína em direção ao filtrado glomerular, e a não reabsorção destas proteínas pelos túbulos proximais. Esse tipo de proteinúria pode ser achado nos casos de glomerulonefrite, amiloidose, síndrome de Fanconi, intoxicação com antibióticos aminoglicosídeos e metais pesados (GRAUER, 2009).

White et al. (1988) demonstraram em cães, alta correlação entre o valor de excreção de proteína urinária em 24 horas e a relação proteína: creatinina (RP/C) de amostras de urinas aleatórias, fundamentando-se no fato de que a creatinina é produzida numa taxa constante, é livremente filtrada pelo glomérulo e não é significativamente secretada nem reabsorvida pelos túbulos renais. Jacob et al. (2003) mostraram que uma RP/C maior que 1,0 em 45 cães com IRC foi associada ao maior risco de desenvolvimento de crise urêmica e morte em comparação com cães cujas RP/C eram menores que 1,0. Estes autores, portanto, concluíram que a determinação da RP/C em cães com IRC de ocorrência natural pode ser usada como valor prognóstico. Atualmente a International Renal Interest Society (IRIS 2006), classificou 
valores da RP/C para cães entre 0 a 0,2 como não proteinúricos, de 0,21 a 0,49 são considerados valores de caráter reservado e quando acima de 0,5 são classificados como proteinúricos; e para gatos valores da RP/C entre 0 a 0,2 como não proteinúricos, de 0,21 a 0,39 são considerados valores de caráter reservado e quando acima de 0,4 são classificados como proteinúricos.

O desfecho de uma proteinúria de elevada intensidade pode ser a Síndrome Nefrótica, caracterizada por hipoalbuminemia, edema, ascite, hipercolesteremia, hipertensão e hipercoagulabilidade (GREGORY, 2005).

A hipertensão arterial no doente renal crônico não está somente relacionada à proteinúria, autores descrevem sua correlação com danos oculares, estudos desenvolvidos por Stiles et al. (1994) descrevem sua ocorrência em gatos e Grauer (2009) descreve sua ocorrência em cães. As lesões oculares características para estes casos são: (1) o estreitamento e perda da tortuosidade das artérias retinianas mais calibrosas, em associação com leve hipertensão arterial, ou vasos dilatados, tortuosos, nos casos de hipertensão arterial mais grave; (2) manchas algodonosas na retina, que são formadas pelo acúmulo de organelas celulares na camada de fibras nervosas da retina, em decorrência de lesões dos axônios; (3) hemorragias; (4) exsudatos; (5) descolamentos da retina; (6) papiledema (ACIERNO; LABATO, 2005). Em um estudo realizado por Silva (2002) com cães hipertensos com doença renal crônica estágios II e III naturalmente adquirida, foram verificadas retinopatias hipertensivas em 4 cães dos 11 cães estudados, as lesões encontradas caracterizavam-se em estágios mais avançados por um aumento da tortuosidade e do calibre dos vasos da retina.

Desordens decorrentes da ação direta do acúmulo de toxinas urêmicas, da hipertensão arterial secundária, e do hiperparatireoidismo secundário renal levam ao aparecimento de sintomas neuromusculares, como head tilt, ataxia, apatia, desorientação, mioclonias, convulsões e coma, com prognóstico reservado (ACIERNO; LABATO, 2005).

\section{DIAGNÓSTICO DA HIPERTENSÃO ARTERIAL}

Em medicina de animais de companhia a hipertensão arterial é diagnosticada através de métodos diretos ou indiretos. O método direto para a mensuração da pressão arterial compreende na canulação arterial direta, geralmente da artéria carótida ou femoral. Em virtude da utilização de equipamentos especiais e devido a necessidad de um procedimento anestésico para a mensuração direta, a indireta é rotineiramente utilizada para o diagnóstico (WEIGNER, 2004). As opções para determinação da pressão arterial pelo método indireto 
envolvem no uso do método oscilométrico, o emprego do doppler vascular e a pletismografia. O método oscilométrico atua na detecção das oscilações na pressão do manguito para determinar a pressão sanguínea sistólica, diastólica e média; já o doppler vascular consiste em detectar o fluxo sanguíneo arterial, sendo uma medida menos onerosa e mais precisa que o método oscilométrico; a pletismografia é um recurso mais recente, que utiliza a radiação infravermelha para determinar a pressão arterial, a precisão desta técnica se assemelha com o método do doppler vascular (WEIGNER, 2004; MUCHA; CAMACHO, 2007).

Para a mensuração da pressão arterial pelo método indireto, é necessário o uso do manguito, que deve ser de tamanho apropriado para obter determinações precisas, desse modo, a largura do manguito deve ser correspondente a $40 \%$ da circunferência do membro. $\mathrm{O}$ paciente deve permanecer em decúbito lateral, para que o membro esteja no mesmo nível do coração. O fluxo sanguíneo mensurado geralmente é nas artérias digitais comuns plantar ou palmar (WEIGNER, 2004).

Independente do método de diagnóstico escolhido para determinação da pressão arterial em cães e gatos é necessário que estes animais passem por um período de adaptação a conduta escolhida. Para a obtenção de um valor preciso e correto, desta forma, é necessário que o animal esteja familiarizado com o ambiente e com a metodologia aplicada, para que fatores como o estresse não induza um falso diagnóstico, assim um ambiente sereno e agradável ao paciente, e um profissional qualificado são estratégias a serem consideradas (ACIERNO; LABATO, 2005).

Embora exista variação nos parâmetros informados na literatura, geralmente são considerados hipertensos animais (cães ou gatos) que apresentem pressão arterial sistólica igual ou superior a 170mmHg. Para o diagnóstico seguro indica-se que a pressão arterial seja aferida em três tempos diferentes, e em cada tempo sejam realizadas sete aferições, sendo posteriormente descartado dois valores limítrofes (maior e menor), permanecendo cinco valores, dos quais se obtêm a média (MORAIS; PEREIRA, 2002; TILLEY, 2009).

A IRIS (2006) caracterizou valores para cães e gatos com doença renal crônica, sendo a pressão arterial sistólica de 130 a $150 \mathrm{mmHg}$ de mínimo risco, de 150 a $160 \mathrm{mmHg}$ de baixo risco, de 160 a $180 \mathrm{mmHg}$ moderado risco e superior a $180 \mathrm{mmHg}$ de alto risco.

Estudo realizado por Stepien (2002) descreve as seguintes manifestações clínicas possíveis em cães e gatos com DRC hipertensos de acordo com o valor da pressão arterial aferido: (1) quando igual ou superior a $170 \mathrm{mmHg}$, nos rins ocorre um maior déficit de seu funcionamento, e a proteinúria pode estar presente; (2) quando igual ou superior a $180 \mathrm{mmHg}$, nos olhos são descritos hemorragia vítrea, edema de retina, descolamento de retina, 
tortuosidade dos vasos da retina, hifema e papiledema, ainda podem ocorrer manifestações neurológicas nestes casos.

Vale ressaltar que caso o paciente apresente sinais neurológicos, descolamento de retina, hemorragias retinianas ou pressão arterial sistólica superior a $250 \mathrm{mmHg}$, estes pacientes são considerados emergenciais, devendo ser instituído o tratamento de crise hipertensiva (MORAIS; PEREIRA, 2002).

\section{TRATAMENTO DA HIPERTENSÃO NO DOENTE RENAL CRÔNICO}

Em pequenos animais com hipertensão arterial e DRC, é frequentemente recomendado o uso de agentes antihipertensivos que promovam a vasodilatação, pois estes fármacos são protetores da função renal, por preservação da TFG (BROWN apud ETTINGER; FELDMAN, 2005). Duas classes de vasodilatadores são consideradas como protetores da função renal, são eles os inibidores da enzima de conversão da angiotensina e os bloqueadores dos canais de cálcio. É sabido que a pressão intraglomerular aumentada promove um aumento na TFG, isto ocorre devido a vasodilatação da arteríola aferente e uma vasoconstrição da arteríola eferente, desse modo ocorre o acréscimo da pressão intraglomerular promovendo os efeitos deletérios nos rins (BROWN apud ETTINGER; FELDMAN, 2005).

O uso de antihipertensivos com efeito vasodilatador, como os inibidores da enzima de conversão da angiotensina (por exemplo: enalapril, benazepril e captopril), promovem uma redução da pressão arterial sistêmica por vasodilatação, por reduzirem os níveis séricos de angiotensina II e também promovem uma vasodilatação da arteríola eferente glomerular, reduzindo a pressão intraglomerular e aumento do fluxo sanguíneo renal, reduzindo a proteinúria, desse modo possuindo um efeito benéfico aos rins no cão doente renal crônico hipertenso, bem como reduzindo o risco de complicações oculares e neurológicas induzidas pela hipertensão (MORAIS; PEREIRA, 2002; ACIERNO; LABATO, 2005; BROWN apud ETTINGER; FELDMAN, 2005).

Outra classe de antihipertensivos com ação de proteção aos rins são os bloqueadores dos canais de cálcio, estes atuam na vasodilatação por interferirem na contração vascular dependente de cálcio. Os dois fármacos mais utilizados deste grupo são o diltiazem e a amlodipina, sendo o bensilato de amlodipina o medicamento seguro e eficaz no tratamento do felino doente renal crônico hipertenso (MORAIS; PEREIRA, 2002; BROWN apud ETTINGER; FELDMAN, 2005; TILLEY, 2009). Segundo Brown; Brown; Jacob et al. (2001) e Acierno e Labato (2005), mais de 50\% dos gatos hipertensos, não respondem a ação dos 
inibidores da enzima conversora da angiotensina como opção em tratamento, pois se acredita que o mecanismo patofisiológico envolvido na hipertensão em gatos, não seja associado somente a ativação da renina, acredita-se que outros fatores estejam envolvidos, ainda não totalmente esclarecidos.

Em gatos, a terapia da hipertensão é iniciada com o bensilato de amlodipina, em cães, a amlodipina não é tão eficaz, e a droga inicial empregada é o vasodilatador do grupo dos inibidores da enzima de conversão da angiotensina. Todos os medicamentos devem ser iniciados com a menor dose, sendo três semanas o tempo máximo necessário para concluir a eficácia do fármaco; caso este medicamento seja parcialmente eficaz, a dose deve ser aumentada ou um segundo fármaco deve ser associado (MORAIS; PEREIRA, 2002).

De acordo com alguns autores (ACIERNO; LABATO, 2005; SNYDER; COOKE apud ETTINGER; FELDMAN, 2005) o bensilato de amlodipina promove uma redução da hipertrofia ventricular, reduz o risco de complicações oculares e neurológicas, em gatos com doença renal crônica e hipertensão.

A alternativa terapêutica em casos de crise hipertensiva para cães e gatos é a utilização da hidralazina oral ou diltiazem oral, estas opções em tratamento são aplicadas principalmente quando não se é possível o monitoramento contínuo da pressão arterial. Caso tenha a disponibilidade de recursos em monitoramento contínuo da pressão arterial o uso do nitropussiato de sódio intravenoso é considerado como o fármaco de escolha (MORAIS; PEREIRA, 2002).

Depois de estabelecido o tratamento adequado ao doente renal com hipertensão, os valores da função renal e da pressão arterial devem ser avaliados periodicamente, para um melhor acompanhamento das condições do paciente (ACIERNO; LABATO, 2005).

A hipertensão arterial sistêmica atua na progressão e evolução da doença renal crônica com maior magnitude, com o comprometimento de outros órgãos, como coração, cérebro, olhos, e os próprios rins, desta forma, a determinação da pressão arterial no momento do diagnóstico no paciente renal crônico é de suma importância, pois estabelecido o diagnóstico de hipertensão arterial e após a introdução da forma terapêutica adequada, ocorre atenuação da progressão da doença renal crônica, com consequente minimização do comprometimento de outros órgãos, bem como aumento e melhoria na qualidade de vida destes pacientes.

\section{REFERÊNCIAS}

ACIERNO, M.J.; LABATO, A. Hypertension in renal disease: diagnosis and treatment. Clinical Techniques in Small Animal Practice, Baton Rouge, v. 20, P. 23-30, 2005. 
BROWN, S. A.; BROWN, C. A.; JACOBS, G. Effects of the angiotensina converting enzyme inhibitor benazepril in cats with induced renal insufficiency. American Journal of

Veterinary Research, v. 62, n. 3, p. 375-383, 2001.

BROWN, S.A. Pathophysiology of systemic hypertension. In: ETTINGER S.J.; FELDMAN E.C. Textbook of veterinary internal medicine. 6th. Philadelphia: W. B. Saunders, 2005, v. 1, cap. 129 , p.472-476.

BARTGES, J.W. HYPERTENSION AND RENAL DISEASE. Veterinary Clinics of North American, Manhatam, v. 26, n. 6, p. 1331-1345, 1996.

COWGILL, L.; KALLET, A. Systemic hypertension. In: KIRK, R. Current Veterinary

Therapy IX. Philadelphia: W. B. Saunders Company, 1986, cap. 12, p. 360-364.

DARKE, P.; BONAGURA, J.D.; KELLY, D.F. DOENÇAS CARDIOVASCULARES. IN: DARKE, P.; BONAGURA, J.D.; KELLY, D.F. Cardiologia Veterinária. São Paulo: Manole, 2000, cap. 21, p. 134-135.

DESIR, G.V. Regulation of blood pressure and cardiovascular function by renalase. Kidney International, New Haven, v. 76, n 04. p. 366-370, 2009.

DESIR, G.V. Renalase deficiency in chronic kidney disease, and its contribution to hypertension and cardiovascular disease. Current Opinion in Nephrology and Hypertension, New Haven, v. 17, p. 181-185, 2008.

FINCO, D.R. Applied physiology of the kidney. In: OSBORNE, C.A.; FINCO, D.R. Canine and Feline Nephrology and Urology. 2 ed. Philadelphia: Williams \& Willkins, 1995, p. 2646.

FROHLICH, E.D. The heart in hypertension. The New England Journal of Medicine. London, v. 328, p. 212-213, 1993.

GRAUER, G.F. et al.; Effects of enalapril versus placebo as a treatment for canine idiopathic glomerulonephritis. Journal of Veterinary Internal Medicine, Fort Collins, v. 14, n. 5, p. 526-533, 2000.

GRAUER, G.F. [2009]. Diagnosis, management of hypertension, proteinúria in dogs with chronic renal disease. DVM Newsmagazine, Clevenland, Ohio-USA April 2009. Disponível em: < http://veterinarynews.dvm360.com/dvm/medicine/diagnosis-management-ofhypertension-proteinuria-i/articlestandard/article/detail/591639? contextcategoryid $=45705$ > acesso em: 10 ago. 2009.

GREGORY, F.G. Early detection of renal damage and disease in dogs and cats. Veterinary Clinical Small Animal, Florida, v. 35, p. 281-296, 2005.

IRIS [2006]. International renal interest society. Staging Chronic Kidney Disease (CDK). Disponível em: <http://www.iris-kidney.com/pdf/iris\%20a4\%20poster.pdf $>$. acesso em: 10 ago. 2009. 
JACOB, F. Clinical evolution of dietary modification for treatment of spontaneous chronic renal failure in dogs. Journal of the American Veterinary Medical Association, St. Paul, n. 220. p. 1163-1170, 2002.

JACOB, F.et al. Association between initial systolic blood pressure and risk of developing a uremic crisis or of dying in dogs with chronic renal failure. Journal of the American Veterinary Medical Association, Minnesota, v. 222, n. 3, p. 322-329, 2003.

KLAG, M.J.et al. Blood pressure and end-stage renal disease in men. The New England Journal of Medicine, Baltimore, v. 334, p. 13-18, 1996.

LONDON, G.M.et al. Cardiac and arterial interactions in end-stage renal disease. Kidney International, London, v. 50, p. 600-608, 1996.

MUCHA, J.C.; CAMACHO, A.A. Determinación de la presión arterial. In: BELEREINAN, G.et al. Afecciones Cardiovasculares em Pequeños Animales. 2.ed. Buenos Aires: InterMédica, 2007, cap. 08, p. 179-183.

MORAIS, H.A.; PEREIRA, P.M. Terapêutica do sistema cardiovascular. In: ANDRADE, S.F. Manual Terapêutica Veterinária. 2. ed. São Paulo: Roca, 2002, Cap.13, p. 265-284.

MUNAGALA, V.K.et al. Ventricular structure and function in aged dogs with renal hypertension. Circulation Research, Dallas, v. 111, p. 1128-1135, 2005.

ORTEGA, T. M.et al. Systemic arterial blood pressure and urine protein/cratinine ratio in dogs with hyperadrenocorticism. Journal of the American Veterinary Medical Association, Davis, v. 15, n. 209, p. 1724-1729, 1996.

POLZIN, D.J.; OSBORNE, C.A.; BARTGES, J.W. Chronic renal failure. In: ETTINGER, S.J.; FELDMAN, E.C. Textbook of veterinary internal medicine. 5 ed. Philadelphia: W. B. Saunders, 2000, Cap.239, p. 1634-1662.

POLZIN, D.J.; OSBORNE, C.A.; ROSS, S. Chronic renal failure. In: ETTINGER, S.J.; FELDMAN, E.C. Textbook of veterinary internal medicine. 6 ed. Philadelphia: W. B. Saunders, 2005, Cap.260, p. 1756-1785.

REGO, A. B. A. S. Microalbuminúria em cães com insuficiência renal crônica: relação com pressão sangüínea sistêmica. 2006. 97f . Dissertação de mestrado em medicina veterinária - Faculdade de Medicina Veterinária e Zootecnia, Universidade de São Paulo.

ROSS, L. Hypertension and chronic renal failure. Seminars in Veterinary Medicine and Surgery (small animal), Bristol, v. 7, n. 3, p. 221-226, 1992.

SCHIFFRIN, E.L.; LIPMAN, M.L.; MANN, J.F.E. Chronic Kidney Disease. Circulation Research, Dallas, v. 116, p. 85-97, 2007.

SCHLAICH, M.P.et al. Sympathetic activation in chronic renal failure. Journal American Society Nephrology, Melbourne, v. 20, p. 933-999, 2009.

SCOTT, A.N.D. Oxidative stress and chronic kidney disease. Veterinary Clinical Small Animal, Athens, v. 38, p. 157-166, 2008. 
SHIMIZU, M. H. M. A N-acetilcisteína atenua a progressão da doença renal crônica. 2005. Ribeirão Preto, 105f. Tese de doutorado em medicina.. Faculdade de Medicina Ribeirão Preto. Departamento de Clínica Médica. Universidade de São Paulo. Ribeirão Preto.

SILVA, S. R. R. Pressão arterial e sua relação com a homeostase de sódio em cães com insuficiência renal crônica. 2002. Jaboticabal, 51f. Dissertação de mestrado em medicina veterinaria, Faculdade de Ciências Agrárias e Veterinárias, Universidade Estadual Paulista, Jaboticabal.

SNYDER, P. S.; COOKE, K. L. Management of hypertension. In: ETTINGER, S.J.; FELDMAN, E.C. Textbook of veterinary internal medicine. 6 ed. Philadelphia: W. B. Saunders, 2005, Cap.219, p. 477-479.

STEPIEN R. L. [2002]. Hypertension in cats and dogs. The 26 th Annual Walthan Diets, Symposion small animal cardiology. Ohio, USA.. Disponível em http://www.vin.com/proceedings/Proceedings.plx?CID=WALTHAMOSU2002\&PID=2989 Acesso em: 27 set. 2009.

STILES, J; POLZIN, D.J.; BISTNER, S.I. The prevalence of retinopathy in cats with systemic hypertension and chronic renal failure or hyperthyroidism. Journal American Animal Hospital Association, London, v. 30, n. 6, p. 564-572, 1994.

STRUBLE, A.L.et al. Systemic hypertension and proteinúria in dogs with diabetes mellitus. Journal of the Amercian Veterinary Medical Association, Davis, v. 213, n. 6, p. 822-825, 1998.

TILLEY, L. [2008]. Diagnosing and treating hypertension (Proceedings): CVC Clevenland, Ohio-USA, Out 2008. Disponível em: < http://veterinarycalendar.dvm360.com/avhc/Medicine/Diagnosing-and-treating-hypertensionProceedings/ArticleStandard/Article/detail/588617 >.Acesso em: 10 ago. 2009.

TOTO, R.D.et al. Strict blood pressure control and progression of renal disease in hypertensive nephroesclerosis. Kidney International, Nürnberg, v. 48, p. 851-589, 1995.

WHITE, V.; FASS, G.; BONE, J. M. Renal pathology and proteinúria determine progression in untreated mild/moderate chronic renal failure. The Quartely Journal of Medicine, Liverpoll v. 67, p. 343-354, 1988.

WEHNER, A.; HARTMANN, K.; HIRSCHBUGER J. Associations between proteinuria, systemic hypertension and glomerular filtration rate in dogs with renal and non-renal diseases. Veterinary Records, Munich v. 162, v. 7, p. 223-224, 2008.

WEIGNER, D. Hipertensão sistêmica. In: LAPPIN, R. M. et al. Segredos em medicina interna de felinos. São Paulo: Artmed, 2004. cap 63, p. 391-396.

$\mathrm{XU}$, J.et al. Renalase is a novel, soluble monoamine oxidase that regulates cardiac function and blood pressure. Journal of Clinical Investigation, New Haven, v. 115, n. 05, p. 1275$1280,2005$. 